\title{
Core-collapse Supernovae as Cosmic Ray Sources
}

\author{
Vikram Dwarkadas* \\ University of Chicago \\ E-mail: vikrameastro.uchicago.edu
}

\section{Alexandre Marcowith}

Universite Montpellier

E-mail: almarcowithegmail.com

\section{Matthieu Renaud}

Universite Montpellier

E-mail: matthieu.renaudeumontpellier.fr

\section{Vincent Tatischeff}

Universite Paris-Sud

E-mail: Vincent.Tatischeffecsnsm.in2p3.fr

\section{Gwenael Giacinti}

Max-Planck-Institut fur Kernphysik

E-mail: Gwenael.Giacinti@mpi-hd.mpg.de

Core-collapse supernovae produce fast shocks which expand into the dense circumstellar medium (CSM) of the stellar progenitor. Cosmic rays (CRs) accelerated at these shocks can induce the growth of electromagnetic fluctuations in the pre-shock medium. Using a self-similar description for the shock evolution, we calculate the growth time-scales of $\mathrm{CR}$ driven instabilities for $\mathrm{SNe}$ in general, and SN 1993J in particular. We find that extended SN shocks can trigger fast intra-day instabilities, strong magnetic field amplification, and CR acceleration. In particular, the nonresonant streaming instability can contribute to about 50 per cent of the magnetic field intensity deduced from radio data. This results in the acceleration of CR particles to energies of 1-10 PeV within a few days after the shock breakout.

36th International Cosmic Ray Conference -ICRC2019-

July 24th - August 1st, 2019

Madison, WI, U.S.A.

\footnotetext{
${ }^{*}$ Speaker.
} 


\section{Introduction}

High-energy cosmic rays (CRs) are likely accelerated in fast shocks produced in very energetic events [1]. CRs above an energy of $10^{17}-10^{18} \mathrm{eV}$ are expected to arise from extragalactic sources. Below this energy the sources are thought to be Galactic, such as young supernova remnants (SNRs). Many of these SNRs have magnetic field strengths much larger than could be expected from shock compression of the interstellar medium magnetic field.

The process of amplification of the magnetic field is unclear. One argument is that magnetic field amplification (MFA) originates from plasma instabilities driven by CR ions [2, 3, 4, 5, 6, 7].

An important argument raised in [2] is that the fastest instability, induced by CR current streaming ahead the shock front, has a growth rate $\Gamma_{\mathrm{g}} \propto n_{0}^{1 / 2} V_{\mathrm{sh}}^{3}$ where $n_{0}$ and $V_{\mathrm{sh}}$ are the ambient gas density and the shock velocity, respectively. Hence, the largest magnetic field fluctuation growth rates produced by energetic particles at an energy $E$ are obtained in dense environments pervaded by fast shocks. Some authors $[8,9,10]$ have therefore pointed to the earliest stages of SN evolution (within months to years of explosion) as possible PeVatron accelerators.

Core-collapse $\mathrm{SNe}$ arise from massive stars, which lose considerable mass during their lifetime. The SNe thus expand into the wind region formed by the progenitor star. For constant wind mass-loss parameters (mass-loss rate and wind velocity) the density of the region drops as $\mathrm{r}^{-2}$, and thus is maximum close in to the star. One possibility therefore would be to search for gamma-ray emission at a very early expansion stage, when the forward shock is interacting with the very dense circumstellar medium (CSM). GeV gamma-rays and neutrinos appear to be the best opportunities to test particle acceleration and $\mathrm{CR}$ production in $\mathrm{SNe}$ [11]. However $\mathrm{GeV}$ photons associated with interaction-powered SNe have not been detected in a Fermi-LAT data search of a sample of 147 SNe of type IIn and Ibn [12]. A search of 45 super-luminous supernovae (SLSNe) with the FermiLAT telescope [13] also did not find any excess $\gamma$-rays at the SLSN positions. A recent study of 10 archival SNe [14], observed with the H.E.S.S. Observatory [15] within a year after explosion, found no significant evidence of $\mathrm{TeV}$ gamma-ray emission from any of the young SNe.

Our goal in this paper is a proper evaluation of the gamma-ray emission during the early phase of blast wave expansion. Following the approach adopted in [16] we derive a general formalism including SN dynamics and wind properties, which can be applied to any SN type where selfsimilar solutions [17] are applicable. We assume that the self-similar solutions are applicable even when some of the SN energy is expended in accelerating particles. This is a reasonable assumption provided that the CR pressure does not exceed $\sim 10 \%$ of the gas pressure [18, 19].

The main hypothesis driving our study is that CR-driven plasma instabilities lead to the magnetic fields deduced from radio monitoring of SNe (see [9] and [20]). Starting from this assumption we adapt the theory of diffusive shock acceleration [21,22] to the case of fast moving forward shocks expanding into the CSM produced by the wind of the SN progenitor star. Within the adopted formalism we discuss the different instabilities that may lead to MFA, and test CR acceleration efficiency at the forward shock for core-collapse SNe. We also include an accurate treatment of the evolution of the CR maximum energy with time.

The results obtained herein are quite general, and applicable to any core-collapse SN whose ejecta density profile and surrounding medium density profile can be described by a power law. Specific calculations are made for the case of SN 1993J. 


\section{Shock dynamics}

The shock radius and velocity are assumed to evolve as a power-law with time. The initial time after the $\mathrm{SN}$ outburst is $t_{0}$ and the corresponding shock radius is $R_{0}$. We have:

$$
R_{\mathrm{sh}}(t)=R_{0} \times\left(\frac{t}{t_{0}}\right)^{m}, V_{\mathrm{sh}}(t)=\frac{R_{0} m}{t_{0}} \times\left(\frac{t}{t_{0}}\right)^{m-1}
$$

We note $V_{0}=R_{0} m / t_{0}$. If the ejecta density of the $\mathrm{SN} \rho_{e j}=A t^{-3} \mathrm{v}^{-k}$, and the surrounding medium density $\rho_{c s}=C r^{-s}$, then a self-similar solution for the shock evolution gives [23]:

$$
R_{\mathrm{sh}}(t)=\beta\left(\frac{\alpha A}{C}\right)^{1 /(k-s)} t^{(k-3) /(k-s)},
$$

\section{Wind density profile}

The wind mass density scales as a power-law with an index $s$ which depends on the mass-loss history of the progenitor. For a steady wind (constant mass-loss rate and wind velocity) $s=2$. The mass density experienced by the forward shock at a time $t$ is, using Eq.(2.1),

$$
\begin{gathered}
\rho_{\mathrm{CSM}}(t)=\rho_{0}\left(\frac{R_{\mathrm{sh}}(t)}{R_{0}}\right)^{-s}=\rho_{0}\left(\frac{t}{t_{0}}\right)^{-m s}, \\
\rho_{0}=\frac{\dot{M}\left(R_{0}\right)}{4 \pi V_{\mathrm{w}}\left(R_{0}\right) R_{0}^{2}} \simeq 1.3 m_{\mathrm{p}} n_{\mathrm{H}, 0},
\end{gathered}
$$

where the factor 1.3 accounts for the presence of a medium containing $90 \% \mathrm{H}$ and $10 \% \mathrm{He}$, and $m_{\mathrm{p}}$ and $n_{\mathrm{H}, 0}$ are the proton mass and hydrogen density at $t_{0}$. We have for the CSM density at $R_{0}$

$$
\rho_{0} \simeq\left[\frac{5.0 \times 10^{13}}{R_{0}^{2}} \mathrm{~g} / \mathrm{cm}^{3}\right] \dot{M}_{-5}\left(R_{0}\right) V_{\mathrm{w}, 10}\left(R_{0}\right)^{-1},
$$

where, the shock radius at $t_{0}$ is expressed in $\mathrm{cm}$, the progenitor mass-loss rate $\dot{M}$ is expressed in units of $10^{-5} M_{\odot} \mathrm{yr}^{-1}$ and the wind asymptotic speed $V_{\mathrm{w}}$ is in units of $10 \mathrm{~km} / \mathrm{s}$. The mass-loss rate is derived at a fixed radius $R_{\text {ref }}=10^{15} \mathrm{~cm}$ (see [24]). The mass-loss rate at $R_{0}$ is by definition given by $\dot{M}\left(R_{0}\right)=\dot{M}\left(R_{\text {ref }}\right)\left(R_{\text {ref }} / R_{0}\right)^{2-s}$. We consider the wind velocity to be constant with the radius.

\section{Magnetic field strength}

The magnetic field strength at the stellar surface obtained by a balance between magnetic field energy density and wind kinetic energy density is:

$$
B_{\mathrm{eq}, 0} \simeq\left[\frac{2.5 \times 10^{13}}{R_{0}} \mathrm{G}\right] \dot{M}_{-5}^{1 / 2} V_{\mathrm{w}, 10}^{1 / 2}
$$

We assume a CSM magnetic field strength proportional to $B_{\text {eq }}$ with

$$
B_{\mathrm{w}}(t) \simeq \varpi B_{\mathrm{eq}, 0}\left(\frac{t}{t_{0}}\right)^{\frac{-m s}{2}}
$$


where we assume the ratio $\varpi=B_{\mathrm{w}}\left(t_{0}\right) / B_{\text {eq, } 0}$ to be in the range $0.1-10$. The time dependence arises from the radial dependence of the wind density as mentioned in section 2 .

In Eq. (4.1) as soon as $R(t) \gg R_{\star}$, the wind magnetic field scales as $1 / R_{\mathrm{sh}}$ which is expected in case of a toroidal geometry. The ambient Alfvén velocity $V_{\mathrm{A}, \mathrm{CSM}}=B_{\mathrm{W}} / \sqrt{4 \pi \rho_{\mathrm{CSM}}}=\varpi V_{\mathrm{w}}$ and the CSM magnetization $\mathscr{M}=\left(V_{\mathrm{A}, \mathrm{CSM}} / c\right)^{2}=\mathscr{M} \simeq\left[\begin{array}{lll}1.1 & 10^{-9}\end{array}\right] \varpi^{2} V_{\mathrm{w}, 10}^{2}$. Considering $\varpi$ to be in the range $0.1-10$ we always obtain $\mathscr{M} \ll 1$ whatever the type of progenitor.

\section{SN 1993J}

Supernova 1993J, at a distance of $3.63 \mathrm{Mpc}$ [25], became the optically brightest SN in the northern hemisphere. It resulted from the explosion of a massive star in a binary system with a progenitor mass ranging in the interval 13-20 $M_{\odot}$ [26]. The star then evolved into a red super-giant (RSG) phase with a mass loss rate of $\sim 10^{-6}$ to $10^{-5} M_{\odot} \mathrm{yr}^{-1}$ and a slow wind $V_{w} \sim 10 \mathrm{~km} / \mathrm{s}$ [27].

\section{Acceleration models}

We adopt a model for particle acceleration at collisionless shocks based on the theory of DSA [21]. The highest energy CRs have an upstream diffusion coefficient $\kappa_{\mathrm{u}}$ which fixes the length scale of the CR precursor $\ell_{\mathrm{u}}=\kappa_{\mathrm{u}} / V_{\mathrm{sh}}$. The timescale to advect the frozen CR-magnetized fluid to the shock front is $T_{\mathrm{adv}, \mathrm{u}}=\frac{\kappa_{\mathrm{u}}}{V_{\mathrm{sh}}^{2}}$. CRs at energies close to $E_{\mathrm{max}}$ stream ahead of the shock and simultaneously generate electromagnetic fluctuations. The upstream diffusion coefficient at these energies can be expressed with respect to the diffusion coefficients parallel and perpendicular to the background wind magnetic field. This coefficient depends on [28]: $\eta$, the ratio of the parallel CR mean free path to CR Larmor radius $R_{\mathrm{L}}$, and $\theta_{\mathrm{B}}$ the magnetic field obliquity. The parallel diffusion coefficient $\kappa_{\|}=\eta R_{\mathrm{L}} v / 3$, where $v$ is the particle speed. $\eta=1$ corresponds to the Bohm diffusion limit. In parallel shocks $\left(\theta_{\mathrm{B}}=0\right) \kappa_{\mathrm{u}}=\kappa_{\|}$while in perpendicular shocks $\left(\theta_{\mathrm{B}}=\pi / 2\right)$ it matches the perpendicular diffusion coefficient, i.e. $\kappa_{\mathrm{u}}=\kappa_{\perp}$. Without considering magnetic field line wandering in the wind turbulent medium we have $\kappa_{\perp}=\kappa_{\|} /\left(1+\eta^{2}\right)$. Hence, if $\eta \gg 1$ diffusion is suppressed in the perpendicular shock case. If the magnetic field in the wind is purely toroidal and weakly turbulent the advection timescale $T_{\mathrm{adv}, \mathrm{u}}$ drops. If the wind medium has some level of turbulence then we can expect to have a diffusion coefficient close to Bohm $(\eta \sim 1)$, and a non negligible portion of the shock in the parallel configuration.

We define as model $\mathrm{P}$ and model $\mathrm{T}$ the two extreme configurations described above. In model $\mathrm{P}$ the wind magnetic field is assumed to be parallel. The advection time in this case is

$$
T_{\mathrm{adv}, \mathrm{u}, \mathrm{P}} \simeq \frac{\eta_{\mathrm{P}} R_{\mathrm{L}} v}{3 V_{\mathrm{sh}}^{2}}
$$

Accounting for some turbulence in the wind medium, we include a contribution due to perturbations in the wind magnetic field, $\delta B_{\mathrm{u}}$, which is assumed to be in equipartition with the mean field strength $B_{\mathrm{w}, 0}: B_{\mathrm{w}}^{2}=\delta B_{\mathrm{u}}^{2}+B_{\mathrm{w}, 0}^{2}$. This turbulence is assumed to be injected at large wind scales, typically the wind termination shock radius, and $\delta B_{\mathrm{u}} \simeq B_{\mathrm{w}, 0}$ at the highest $\mathrm{CR}$ energies.

Using Eq. (4.1) for the wind mean magnetic field, and the proton Larmor radius $R_{\mathrm{L}} \simeq E / e B_{\mathrm{w}}$ for a $1 \mathrm{PeV}$ particle as $R_{\mathrm{L}} \simeq 3.310^{12} E_{P e V} B_{\mathrm{w}, \mathrm{G}}^{-1} \mathrm{~cm}$ we find an advection time in seconds 


$$
T_{\mathrm{adv}, \mathrm{u}, \mathrm{P}} \simeq\left[\frac{1.310^{9} \eta_{\mathrm{P}} R_{0, \mathrm{~cm}}}{V_{0, \mathrm{~cm} / \mathrm{s}}^{2} \varpi} \mathrm{s}\right] \times \frac{E_{\mathrm{PeV}}}{\dot{M}_{-5}^{1 / 2} V_{w, 10}^{1 / 2}}\left(\frac{t}{t_{0}}\right)^{2(1-m)+m \frac{s}{2}} .
$$

In model $\mathrm{T}$ the wind mean magnetic field is assumed to be toroidal and weakly perturbed with fluctuations of strength $\delta B_{\mathrm{u}, \mathrm{w}}<B_{\mathrm{w}, 0} \simeq B_{\mathrm{w}}$. The advection time is in this case

$$
T_{\mathrm{adv}, \mathrm{u}, \mathrm{T}} \simeq \frac{R_{\mathrm{L}} v}{3 \eta_{\mathrm{T}} V_{\mathrm{sh}}^{2}} .
$$

For the parameters adopted for SN 1993J we have $T_{\mathrm{adv}, \mathrm{u}, \mathrm{T}} \simeq(0.24$ day $) \times\left(1 / \eta_{\mathrm{T}} \varpi\right) \mathrm{E}_{\mathrm{PeV}} \mathrm{t}_{\mathrm{d}}^{1.17}$ and $T_{\mathrm{adv}, \mathrm{u}, \mathrm{P}} \simeq(0.24$ day $) \times\left(\eta_{\mathrm{P}} / \varpi\right) \mathrm{E}_{\mathrm{PeV}} \mathrm{t}_{\mathrm{d}}^{1.17}$, where $t_{\mathrm{d}}$ is the time in days after the SN explosion.

We can deduce the acceleration timescale from the above estimates

$$
T_{\text {acc }, \mathrm{P}}=g(r) T_{\mathrm{adv}, \mathrm{u}, \mathrm{P}}=g(r) \frac{\kappa_{u}}{V_{\mathrm{sh}}^{2}}
$$

where $g(r)=3 r /(r-1) \times\left(1+\kappa_{d} r / \kappa_{u}\right)$ depends on the shock compression ratio $r$ and on the ratio of the downstream to upstream diffusion coefficients. The ratio $\kappa_{\mathrm{d}} / \kappa_{\mathrm{u}}$ depends on the magnetic field obliquity and on the shock compression ratio $r$. We have $\kappa_{\mathrm{d}} / \kappa_{\mathrm{u}}=r_{\mathrm{B}}^{-1}$ with $r_{\mathrm{B}}=B_{\mathrm{d}} / B_{\mathrm{u}}$ is the ratio of magnetic fields in the postshock region and in the wind and $g(r)=3 r /(r-1) \times\left(1+r / r_{\mathrm{B}}\right)$. In the model $\mathrm{P}$, we have $r_{\mathrm{B}} \simeq 1$ and $g(r)=3 r(r+1) /(r-1)$. In the model T the magnetic field is weakly perturbed and perpendicular to the shock normal and $\kappa_{\mathrm{d}} / \kappa_{\mathrm{u}}=r^{-1}$ and $g(r)=6 r /(r-1)$.

\subsection{Magnetic Field Amplification}

Various CR driven instabilities may operate at the SN forward shock, generating magnetic field fluctuations necessary for the DSA process to operate at a high efficiency. These include (1) Bell non-resonant streaming instability [2]: the streaming of CRs ahead of the shock front induces a return current in the background plasma, which triggers magnetic fluctuations at scales $\ell \ll R_{\mathrm{L}}$, where $R_{\mathrm{L}}$ is the Larmor radius of the CRs producing the current. This instability is non-resonant and can be treated using a modified MHD model [2, 29, 3]. (2) Resonant streaming instability [6]: The streaming of CRs faster than the local Alfvén speed is known to produce long-wavelength modes at scales $\ell \sim R_{\mathrm{L}}$. (3) Filamentation instability [30]: Cosmic rays form filamentary structures in the precursors of supernova remnant shocks due to their self-generated magnetic fields, which results in the growth of a long-wavelength instability. (4) Long oblique modes [7]: The presence of turbulence at scales shorter than the CR gyroradius enhances the growth of modes with scales longer than the gyroradius for particular polarizations. Complete details can be found in [31].

Figure 1 plots the advection time and the different growth timescales for model $\mathrm{P}$ for particles with energies of $1 \mathrm{PeV}$, for the case of SN 1993J. At all times non-resonant modes can grow. Large scale modes can be produced by the filamentation instability. The oblique mode instability and the resonant streaming instability have growth timescales larger by factors of $\sim 2.5$ and $\sim 15$ compared to the advection time, and can not grow for this set of parameters. However, these timescales drop more rapidly with time and at some stage can become shorter than the advection time, competing with the filamentation instability to produce long-wavelength perturbations. 


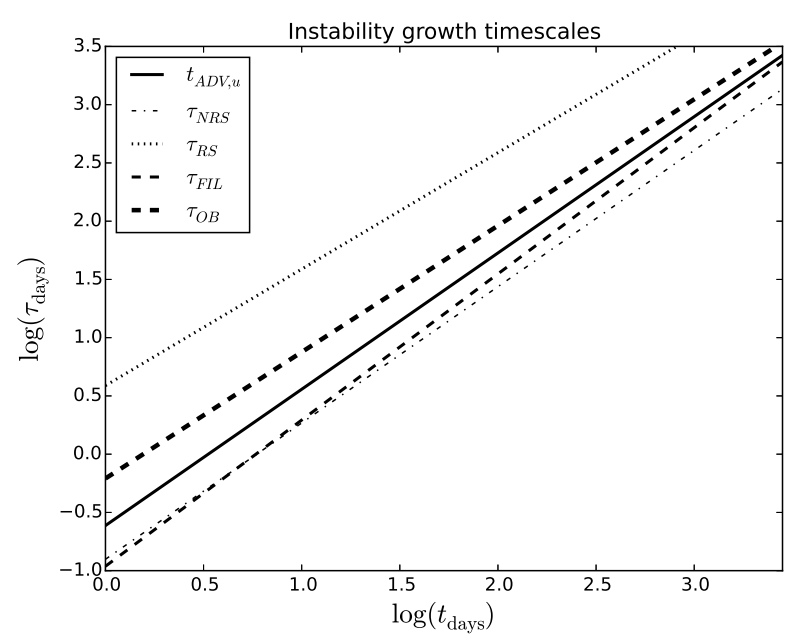

Figure 1: Main instability growth timescales as a function of the time in days for the fiducial case SN 1993. We have assumed $\eta=\varpi=1, E=1 \mathrm{PeV}, \phi=14, \xi_{\mathrm{CR}}=0.05$.

\section{Maximum cosmic ray energies}

The maximum CR (hadronic) energy (Figure 2) is fixed by five different processes: (1) Age limitation, (2) Finite spatial extent of the shock, (3) Generated current limitation, (4) Nuclear interaction losses, and (5) Adiabatic losses.

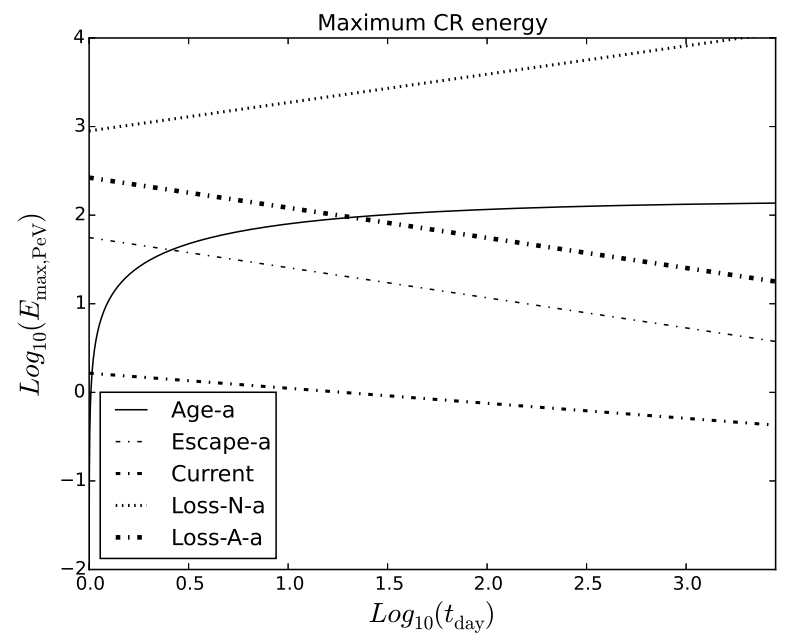

Figure 2: Maximum CR energy limits in $\mathrm{PeV}$ units for the model $\mathrm{P}$ as a function of time after shock breakout

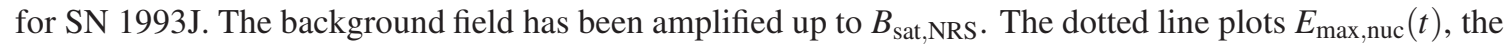
large dot-dashed line plots $E_{\text {max,adi }}(t)$, the intermediate dot-dashed line plots $E_{\max , \text { cur }}(t)$, the small dot-dashed line plot $E_{\max , \text { esc }}(t)$, the solid line plots $E_{\max , \text { age }}(t)$ We use: $\varpi=1, \eta=1, \mathscr{N}=5, \phi=14, \bar{\sigma}_{\mathrm{pp}}=1.87$.

\section{Discussion}

We have shown that $\mathrm{SNe}$ can produce particles up to multi-PeV energies via the combination 
of fast shocks, a high density CSM produced by stellar winds, and low wind magnetization. Assuming that the background magnetic field has a turbulent component, instabilities driven by the acceleration process can grow over intra-day timescales. This model is applied to SN 1993J.

Acknowledgements: VVD is supported by NASA ADAP grant NNX14AR63G, and by the FACCTS program. This work is supported by the ANR-14-CE33-0019 MACH project.

\section{References}

[1] A. R. Bell, The acceleration of cosmic rays in shock fronts. I, MNRAS 182 (Jan., 1978) 147-156.

[2] A. R. Bell, Turbulent amplification of magnetic field and diffusive shock acceleration of cosmic rays, MNRAS 353 (Sept., 2004) 550-558.

[3] G. Pelletier, M. Lemoine and A. Marcowith, Turbulence and particle acceleration in collisionless supernovae remnant shocks. I. Anisotropic spectra solutions, A\&A. 453 (July, 2006) 181-191, [astro-ph/0603461].

[4] A. Marcowith, M. Lemoine and G. Pelletier, Turbulence and particle acceleration in collisionless supernovae remnant shocks. II. Cosmic-ray transport, A\&A. 453 (July, 2006) 193-202, [astro-ph/0603462].

[5] V. N. Zirakashvili, V. S. Ptuskin and H. J. Völk, Modeling Bell's Nonresonant Cosmic-Ray Instability, ApJ 678 (May, 2008) 255-261, [0 801 . 4486 ].

[6] E. Amato and P. Blasi, A kinetic approach to cosmic-ray-induced streaming instability at supernova shocks, MNRAS 392 (Feb., 2009) 1591-1600, [0 806.1223 ].

[7] A. M. Bykov, S. M. Osipov and D. C. Ellison, Cosmic ray current driven turbulence in shocks with efficient particle acceleration: the oblique, long-wavelength mode instability, MNRAS $\mathbf{4 1 0}$ (Jan., 2011) 39-52, [1010.0408].

[8] K. M. Schure and A. R. Bell, Cosmic ray acceleration in young supernova remnants, MNRAS 435 (Oct., 2013) 1174-1185, [1307.6575].

[9] A. Marcowith, M. Renaud, V. Dwarkadas and V. Tatischeff, Cosmic-ray acceleration and gamma-ray signals from radio supernova, Nuclear Physics B Proceedings Supplements 256 (Nov., 2014) 94-100, [1409.3670].

[10] M. Cardillo, E. Amato and P. Blasi, On the cosmic ray spectrum from type II supernovae expanding in their red giant presupernova wind, Astroparticle Physics 69 (Sept., 2015) 1-10, [1503. 03001 ].

[11] K. Murase, T. A. Thompson and E. O. Ofek, Probing cosmic ray ion acceleration with radio-submm and gamma-ray emission from interaction-powered supernovae, MNRAS 440 (May, 2014) 2528-2543, [1311.6778].

[12] M. Ackermann, I. Arcavi, L. Baldini, J. Ballet, G. Barbiellini, D. Bastieri et al., Search for Early Gamma-ray Production in Supernovae Located in a Dense Circumstellar Medium with the Fermi LAT, ApJ 807 (July, 2015) 169, [1506.01647].

[13] N. Renault-Tinacci, K. Kotera, A. Neronov and S. Ando, Search for gamma-ray emission from super-luminous supernovae with the Fermi-LAT, ArXiv e-prints (Aug., 2017), [1708.08971].

[14] H. E. S. S. Collaboration, H. Abdalla, F. Aharonian, F. Ait Benkhali, E. O. Angüner, M. Arakawa et al., Upper limits on very-high-energy gamma-ray emission from core-collapse supernovae observed with H.E.S.S., A\&A. 626 (June, 2019) A57, [1904 . 10526 ]. 
[15] H.E.S.S. Collaboration, A. Abramowski, F. Aharonian, F. Ait Benkhali, A. G. Akhperjanian, E. O. Angüner et al., The exceptionally powerful TeV $\gamma$-ray emitters in the Large Magellanic Cloud, Science 347 (Jan., 2015) 406-412, [1501.06578].

[16] V. V. Dwarkadas, Exploring the $\gamma$-ray emissivity of young supernova remnants - I. Hadronic emission, MNRAS 434 (Oct., 2013) 3368-3377, [1307 . 4414].

[17] R. A. Chevalier, Self-similar solutions for the interaction of stellar ejecta with an external medium, ApJ 258 (July, 1982) 790-797.

[18] R. A. Chevalier, Blast waves with cosmic-ray pressure, ApJ 272 (Sept., 1983) 765-772.

[19] H. Kang and D. Ryu, Diffusive Shock Acceleration in Test-particle Regime, ApJ 721 (Sept., 2010) 886-892, [1008.0429].

[20] A. M. Bykov, D. C. Ellison, A. Marcowith and S. M. Osipov, Cosmic Ray Production in Supernovae, Space Sci. Rev. 214 (Feb., 2018) 41, [1801 . 08890].

[21] L. O. Drury, An introduction to the theory of diffusive shock acceleration of energetic particles in tenuous plasmas, Reports on Progress in Physics 46 (Aug., 1983) 973-1027.

[22] E. G. Berezhko and D. C. Ellison, A Simple Model of Nonlinear Diffusive Shock Acceleration, ApJ 526 (Nov., 1999) 385-399.

[23] R. A. Chevalier and C. Fransson, Emission from circumstellar interaction in normal Type II supernovae, ApJ 420 (Jan., 1994) 268-285.

[24] C. Fransson, P. Lundqvist and R. A. Chevalier, Circumstellar Interaction in SN 1993J, ApJ 461 (Apr., 1996) 993.

[25] W. L. Freedman, B. F. Madore, B. K. Gibson, L. Ferrarese, D. D. Kelson, S. Sakai et al., Final Results from the Hubble Space Telescope Key Project to Measure the Hubble Constant, ApJ 553 (May, 2001) 47-72, [astro-ph/0012376].

[26] J. R. Maund, S. J. Smartt, R. P. Kudritzki, P. Podsiadlowski and G. F. Gilmore, The massive binary companion star to the progenitor of supernova 1993J, Nature 427 (Jan., 2004) 129-131, [astro-ph/ 0401090$]$.

[27] V. Tatischeff, Radio emission and nonlinear diffusive shock acceleration of cosmic rays in the supernova SN 1993J, A\&A. 499 (May, 2009) 191-213, [0903.2944].

[28] J. R. Jokipii, Rate of energy gain and maximum energy in diffusive shock acceleration, ApJ 313 (Feb., 1987) 842-846.

[29] A. R. Bell, The interaction of cosmic rays and magnetized plasma, MNRAS 358 (Mar., 2005) 181-187.

[30] B. Reville and A. R. Bell, A filamentation instability for streaming cosmic rays, MNRAS 419 (Jan., 2012) 2433-2440, [1109.5690].

[31] A. Marcowith, V. V. Dwarkadas, M. Renaud, V. Tatischeff and G. Giacinti, Core-collapse supernovae as cosmic ray sources, MNRAS 479 (Oct., 2018) 4470-4485, [18 06.0970 0]. 